\title{
ON THE CAUSTIC BY REFLECTION AT A CIRCLE.
}

[From the Cambridge and Dublin Mathematical Journal, vol. II. (1847), pp. 128-130.]

THE following solution of the problem is that given by M. de St-Laurent (Annales de Gergonne, t. xvII. [1826] pp. 128-134); the process of elimination is somewhat different.

The centre of the circle being taken for the origin, let $k$ be its radius; $a, b$ the coordinates of the luminous point; $\xi, \eta$ those of the point at which the reflection takes place; $x, y$ those of any point in the reflected ray: we have in the first place

$$
\xi^{2}+\eta^{2}=k^{2}
$$

There is no difficulty in finding the equation of the reflected ${ }^{2}{ }^{1}$; this is

$$
(b \xi-a \eta)\left(\xi x+\eta y-k^{2}\right)+(y \xi-x \eta)\left(a \xi+b \eta-k^{2}\right)=0
$$

1 To do this in the simplest way, write

$$
\rho^{2}=(\xi-x)^{2}+(\eta-y)^{2}, \quad \sigma^{2}=(\xi-a)^{2}+(\eta-b)^{2},
$$

then, by the condition of reflection,

$$
\rho+\sigma=\min .,
$$

$\rho, \sigma$ being considered as functions of the variables $\xi$, $\eta$, which are connected by the equation (1). Hence

or, eliminating $\lambda$,

$$
\begin{aligned}
& \frac{\xi-x}{\rho}+\frac{\xi-a}{\sigma}+\lambda \xi=0 \\
& \frac{\eta-y}{\rho}+\frac{\eta-b}{\sigma}+\lambda \eta=0
\end{aligned}
$$

$$
\frac{\eta x-\xi y}{\rho}+\frac{\eta a-\xi b}{\sigma}=0,
$$

whence

$$
(\eta x-\xi y)^{2}\left[(\xi-a)^{2}+(\eta-b)^{2}\right]=(\eta a-\xi b)^{2}\left[(\xi-x)^{2}+(\eta-y)^{2}\right] .
$$

This may be written $\quad\{(\eta x-\xi y)(\xi-a)-(\eta a-\xi b)(\xi-x)\}[(\eta x-\xi y)(\xi-a)+(\eta a-\xi b)(\xi-x)]$

$$
+\{(\eta x-\xi y)(\eta-b)-(\eta a-\xi b)(\eta-y)\}[(\eta x-\xi y)(\eta-b)+(\eta a-\xi b)(\eta-y)]=0 ;
$$

the factors in \{\} reduce themselves respectively to $\xi P$ and $\eta P$, where $P=\xi(b-y)-\eta(a-x)+a y-b x$; omitting the factor $P$, (which equated to zero, is the equation of the line through $(a, b)$ and $(\xi$, $\eta)$,) and replacing $\xi(\xi-a)+\eta(\eta-b)$ and $\xi(\xi-x)+\eta(\eta-y)$ by $k^{2}-a \xi-b \eta$ and $k^{2}-\xi x-\eta y$, respectively, we have the equation given above.

C. 
or, arranging the terms in a more convenient order,.

$$
(b x+a y)\left(\xi^{2}-\eta^{2}\right)+2(b y-a x) \xi \eta-k^{2}(b+y) \xi+k^{2}(a+x) \eta=0
$$

Hence, considering $\xi, \eta$ as indeterminate parameters connected by the equation (1), the locus of the curve generated by the continued intersections of the lines (2) will be found by eliminating $\xi, \eta, \lambda$ from these equations and the system

$$
\begin{aligned}
& \xi[\lambda+2(b x+a y)]+\eta[2(b y-a x)]-k^{2}(b+y)=0 \\
& \xi[2(b y-a x)]+\eta[\lambda-2(b x+a y)]+k^{2}(a+x)=0
\end{aligned}
$$

and from these, multiplying by $\xi$, $\eta$, adding and reducing by (2), we have

$$
-\xi(b+y)+\eta(a+x)-\lambda=0 .
$$

which replaces the equation (2) or $\left(2^{\prime}\right)$. Thus the equations from which $\xi, \eta, \lambda$ are to be eliminated are (1), (3), (4), (5).

From (3), (4), (5), by the elimination of $\xi$, $\eta$, we have

$$
\begin{aligned}
-\lambda\left\{\lambda^{2}\right. & \left.-4(b x+a y)^{2}\right\}-4 k^{2}(b y-a x)(a+x)(b+y) \\
& -k^{2}(a+x)^{2}[\lambda+2(b x+a y)] \\
& -k^{2}(b+y)^{2}[\lambda-2(b x+a y)] \\
& +4 \lambda(b y-a x)^{2}=0 \ldots \ldots \ldots \ldots \ldots \ldots \ldots \ldots \ldots \ldots \ldots \ldots \ldots \ldots \ldots \ldots \ldots \ldots
\end{aligned}
$$

or, reducing,

$$
\begin{array}{r}
-\lambda^{3}+\lambda\left\{4\left(a^{2}+b^{2}\right)\left(x^{2}+y^{2}\right)-k^{2}\left[(a+x)^{2}+(b+y)^{2}\right]\right\} \\
-2 k^{2}(b x-a y)\left(x^{2}+y^{2}-a^{2}-b^{2}\right)=0 \quad \ldots \ldots \ldots \ldots
\end{array}
$$

which may be represented by

$$
-\lambda^{3}+\lambda Q-2 R=0
$$

Again, from the equations (4), (3), transposing the last terms and adding the squares, also reducing by (1),

$$
\begin{aligned}
k^{4}\left[(a+x)^{2}\right. & \left.+(b+y)^{2}\right]=k^{2} \lambda^{2}+4 k^{2}\left(a^{2}+b^{2}\right)\left(x^{2}+y^{2}\right) \\
& +4 \lambda\left[\left(\xi^{2}-\eta^{2}\right)(b x+a y)+2 \xi \eta(b y-a x)\right]
\end{aligned}
$$

but from the same equations, multiplying by $\xi, \eta$ and adding, also reducing by (1),

$$
k^{2} \lambda+2(b x+a y)\left(\xi^{2}-\eta^{2}\right)+4 \xi \eta(b y-a x)+k^{2}[-\xi(b+y)+\eta(a+x)]=0 .
$$

or reducing by (5) and dividing by two,

$$
k^{2} \lambda+(b x+a y)\left(\xi^{2}-\eta^{2}\right)+2 \xi \eta(b y-a x)=0
$$


Using this to reduce (8),

$$
k^{2}\left[(a+x)^{2}+(b+y)^{2}\right]=4\left(a^{2}+b^{2}\right)\left(x^{2}+y^{2}\right)+3 \lambda^{2}
$$

or, from the value of $P$,

$$
-3 \lambda^{2}+Q=0
$$

which singularly enough is the derived equation of $\left(7^{\prime}\right)$ with respect to $\lambda$ : so that the equation of the curve is obtained by expressing that two of the roots of the equation $\left(7^{\prime}\right)$ are equal. Multiplying (12) by $\lambda$ and reducing by $\left(7^{\prime}\right)$,

$$
-\lambda Q+3 R=0
$$

or, combining this with (12),

$$
27 R^{2}-Q^{3}=0
$$

whence, replacing $R, Q$ by their values, we find

$$
27 k^{4}(b x-a y)^{2}\left(x^{2}+y^{2}-a^{2}-b^{2}\right)^{2}-\left\{4\left(a^{2}+b^{2}\right)\left(x^{2}+y^{2}\right)-k^{2}\left[(a+x)^{2}+(b+y)^{2}\right]\right\}^{3}=0,
$$

the equation of M. de St-Laurent. 